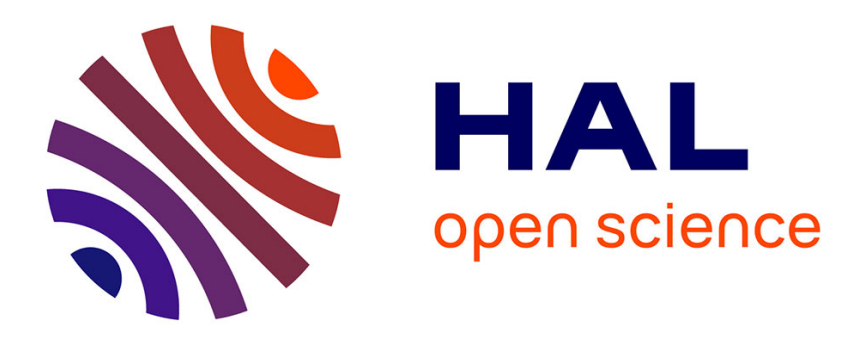

\title{
One-dimensional incommensurate structures at finite temperatures
}

\author{
S.E. Burkov, A.L. Talapov
}

\section{To cite this version:}

S.E. Burkov, A.L. Talapov. One-dimensional incommensurate structures at finite temperatures. Journal de Physique Lettres, 1980, 41 (16), pp.387-390. 10.1051/jphyslet:019800041016038700 . jpa00231804

\section{HAL Id: jpa-00231804 https://hal.science/jpa-00231804}

Submitted on 1 Jan 1980

HAL is a multi-disciplinary open access archive for the deposit and dissemination of scientific research documents, whether they are published or not. The documents may come from teaching and research institutions in France or abroad, or from public or private research centers.
L'archive ouverte pluridisciplinaire HAL, est destinée au dépôt et à la diffusion de documents scientifiques de niveau recherche, publiés ou non, émanant des établissements d'enseignement et de recherche français ou étrangers, des laboratoires publics ou privés. 


\title{
One-dimensional incommensurate structures at finite temperatures
}

\author{
S. E. Burkov and A. L. Talapov \\ Landau Institute for Theoretical Physics, Vorobjevskoe Shosse 2, 117940, Moscow, U.S.S.R.
}

(Reçu le 21 mai 1980, accepté le 23 juin 1980)

\begin{abstract}
Résumé. - On discute les propriétés thermodynamiques des systèmes unidimensionnels d'atomes adsorbés pour tout potentiel périodique du substrat. On obtient les formules exactes pour l'énergie libre et les facteurs de structure en fonction de la température et de la différence des périodes initiales du réseau des atomes adsorbés et du substrat.
\end{abstract}

Abstract. - The problem of one-dimensional systems of adsorbed atoms at finite temperatures is considered. For any form of the periodic potential of a substrate and for any value of the initial incommensurate wave vector the exact formulae for the free energy and for the structural factor of scattering are obtained.

Recently properties of the systems formed by atoms adsorbed with different surfaces have been extensively studied. A great number of experimental research has been devoted to the transition of such structures from a commensurate to an incommensurate phase $[1,2,3]$. These transitions were theoretically studied in one-dimensional systems at zero temperatures in $[4,5,6]$. The corresponding quantum problem was examined by Luther, Timonen and Pokrovsky [7]. Two-dimensional structures were also investigated $[8,9]$.

Experimental data show that both commensurate and incommensurate quasi-one-dimensional structures are observed at finite temperatures [10]. However it is known that phase transitions are impossible in one-dimensional systems. Thus one-dimensional systems must always be incommensurate. In this work we show that for some values of the parameters the deviation from commensuracy proves to be exponentially small at low temperatures. We give exact formulae for the dependence of free energy, order parameter and neutron scattering cross-section on the initial incommensuracy and temperature. Numerical results for the free energy have been obtained in [11] for a particular form of the substrate potential.

We consider the following model : the system consists of atoms interacting with each other and with the periodic substrate potential. This system is described with the Hamiltonian :

$$
\mathscr{H}=\int_{0}^{L}\left[\frac{1}{2}\left(\frac{\mathrm{d} \varphi}{\mathrm{d} x}\right)^{2}-h \frac{\mathrm{d} \varphi}{\mathrm{d} x}+V(\varphi)\right] \mathrm{d} x
$$

$V(\varphi)$ - the external potential of the period $2 \pi$, $\varphi(x)$ - displacement of atoms from minima of $V(\varphi)$. In the absence of the potential of the substrate $V$ the chain of atoms would have the period $2 \pi(1+h)$, $h \ll 1$.

At zero temperature a phase transition takes place at $h=h_{\mathrm{c}}$,

$$
h_{\mathrm{c}}=\frac{1}{2 \pi} \int_{0}^{2 \pi} \sqrt{2\left(V(x)-V_{\min }\right)} \mathrm{d} x .
$$

If $h<h_{\mathrm{c}}$ atoms are positioned at the minima of the potential $V$, if $h>h_{\mathrm{c}}$ they form a chain with a period greater than $2 \pi$.

The order parameter is the mean derivative $\left\langle\frac{\mathrm{d} \varphi}{\mathrm{d} x}\right\rangle$. In the incommensurate phase it tends to zero as $\left|\ln \left(h-h_{\mathrm{c}}\right)\right|^{-1}[6]$.

At finite temperature the problem can be solved exactly by means of reduction of the path integral for free energy $F$ per unit length

$$
\exp \left(-\frac{F L}{T}\right)=\int \mathfrak{D} \varphi(x) \exp \left(-\frac{\mathscr{H}[\varphi(x)]}{T}\right)
$$

to the Schroedinger equation with the imaginary time and with the Hamiltonian :

$$
H=-\frac{T^{2}}{2} \frac{\mathrm{d}^{2}}{\mathrm{~d} \varphi^{2}}+h T \frac{\mathrm{d}}{\mathrm{d} \varphi}+V(\varphi)-\frac{h^{2}}{2} .
$$

The procedure of this kind was applied in $[11,12]$ and some other works. The wave function $\Psi(\varphi, x)$ 
describes the probability that the atom at the point $x$ is displaced by the value $\varphi$. The functions $\psi(\varphi)$ must be limited at infinity. The minimal eigenvalue $E_{0}$ of the operator $H$ is well-known to be equal to the free energy $F$ per unit length of the chain. The order parameter $\left\langle\varphi^{\prime}\right\rangle$ is $-\frac{\partial E_{0}}{\partial h}$.

The temperature $T$ in the Hamiltonian (4) plays the role of Plank's constant and therefore the Schroedinger equation can be solved at small $T$ by means of the WKB approximation. An analogous approach was used in [11] for incommensuracies $h$ which are far from $h_{\mathrm{c}}$, i.e. far from the transition point at $T=0$.

Replacing $\psi=\exp (h \varphi / T) \Phi$ in (4) we come to :

$$
-\frac{T^{2}}{2} \Phi^{\prime \prime}+V(\varphi) \Phi=E \Phi
$$

Let us consider (5) on the segment $[-\pi, \pi]$, putting $\varphi=0$ at the minimum of the potential $V(\varphi)$. At $|\varphi| \ll 1$ one can see, that $V=V_{\min }+\frac{1}{2} \omega^{2} \varphi^{2}$ and the fundamental solutions of (5) are :

$$
\Phi_{1,2}=\mathrm{e}^{-y^{2} / 2} \int_{0}^{\infty} \mathrm{e}^{ \pm y t-t^{2} / 4} t^{m-1} \mathrm{~d} t
$$

where we denote

$$
\begin{aligned}
& y=(\omega / T)^{1 / 2} \varphi, \\
& m=\frac{1}{2}-\left(E-V_{\min }\right) / T \omega, \quad(\operatorname{Re} m>0) .
\end{aligned}
$$

In the region $(T / \omega)^{1 / 2} \ll \varphi \leqslant \pi$ the solutions can be written as

$$
\begin{aligned}
\Phi_{1,2} \sim & \frac{1}{\sqrt[4]{2(V(\varphi)-E)}} \times \\
& \quad \times \exp \left[ \pm \frac{1}{T} \int_{\varphi_{0}}^{\varphi} \sqrt{2(V(x)-E)} \mathrm{d} x\right]
\end{aligned}
$$

where $\varphi_{0}$ is an arbitrary point of the real axis, such as $(T / \omega)^{1 / 2} \ll \varphi_{0} \ll 1$. Matching solutions (6) and (7) in the region $\varphi_{0}<\varphi \ll 1$ we find the fundamental solutions of equation (5) on $[-\pi, \pi]$. Then we construct their linear combination satisfying the boundary conditions. This procedure gives eigenvalues $E(q)$ of the operator $H$ as a function of the wave vector $q$ :

$$
2 \operatorname{ch}\left[\frac{2 \pi}{T}(h-i q T)\right] \frac{\Gamma(m)}{\sqrt{\pi} 2^{m}}\left(\frac{\omega}{T} \varphi_{0}^{2}\right)^{1 / 2-m} \exp \left(-\frac{\omega}{T} \varphi_{0}^{2}\right) \exp \left[-\frac{2}{T} \int_{\varphi_{0}}^{\pi} \sqrt{2(V(x)-E)} \mathrm{d} x\right]=1
$$

The operator $H$ is not Hermitian so its eigenvalues $E$ and the corresponding values of the parameter $m$ are complex. Formula (8) has been derived when $\operatorname{Re} m>0$. However, using analytical continuation it is easy to see that it is valid even at $\operatorname{Re} m<0$. $E$ obtained from (8) does not depend on $\varphi_{0}$. The ground state energy $E_{0}$ corresponds to $q=0$ and is given by the equation :

$$
\begin{aligned}
2 \operatorname{ch} & \left(\frac{2 \pi h}{T}\right) \frac{\Gamma(m)}{\sqrt{2 \pi}}\left(\frac{\mathrm{e}}{|m-1 / 2|}\right)^{m-1 / 2} \times \\
& \times \exp \left[-\frac{1}{T} \int \sqrt{2(V(x)-E)} \mathrm{d} x\right]=1 .
\end{aligned}
$$

The integral is taken between the turning points if $m<1 / 2$ or over period when $m>1 / 2$. Equation (9) also describes other real eigenvalues of $H$. Putting $m=1 / 2, T=0$ in (9) and using (2) we find that equation (9) will be $h=h_{\mathrm{c}}$. It means that the point $m=1 / 2$ corresponds to the second type phase transition at $T=0$ but the free energy $F$ given by equations (8) or (9) has no singularities either at this or any other point.

The deviation of $F$ from its value at $h=0$ is exponentially small at $h \ll T$

$$
F(h)-F(0) \sim \exp \left[\frac{2 \pi\left(h-h_{\mathrm{c}}\right)}{T}\right]
$$

and when $h-h_{\mathrm{c}} \gg T$ it coincides with the energy at zero temperature [6] :

$$
\int_{0}^{2 \pi} \sqrt{2(V(x)-E)} \mathrm{d} x=2 \pi h .
$$

The order parameter is given by the equation $\left\langle\varphi^{\prime}\right\rangle=-\frac{\partial E_{0}}{\partial h}$ and is equal to

$$
\begin{aligned}
\left\langle\frac{\mathrm{d} \varphi}{\mathrm{d} x}\right\rangle=2 \pi & {\left[\int \frac{\mathrm{d} x}{\sqrt{2(V(x)-E)}}+\right.} \\
& \left.+\frac{1}{\omega}\left(\ln \left|m-\frac{1}{2}\right|-\frac{\Gamma^{\prime}(m)}{\Gamma(m)}\right)\right]^{-1}
\end{aligned}
$$

the integration is performed in the same limits as in (9). It follows from (12) that at $h \ll h_{\text {c }}$,

$$
\left\langle\varphi^{\prime}\right\rangle \sim \exp \left[2 \pi\left(h-h_{\mathrm{c}}\right) / T\right],
$$

and at $h-h_{\mathrm{c}} \gg T$,

where

$$
\left\langle\varphi^{\prime}\right\rangle=2 \pi / K(E)
$$

$$
K(E)=\int_{0}^{2 \pi} \frac{\mathrm{d} x}{\sqrt{2(V(x)-E)}} .
$$

This is in agreement with the result for $T=0$ [6]. 
Thus we see that in spite of the absence of the phase transition the order parameter varies sharply in the narrow vicinity of $h_{\mathrm{c}}$ (by width $\sim T$ ).

Let us now investigate the picture of neutron scattering in these structures. The structural factor $S(Q)$ is

$$
S(Q)=\sum_{x} G_{Q}(x) \mathrm{e}^{i Q x}
$$

we have denoted :

$$
G_{Q}(x)=\mathrm{e}^{-i Q h x}\left\langle\mathrm{e}^{i Q(\varphi(x)-\varphi(0))}\right\rangle .
$$

The averaging in this formula is performed with the weight $\exp \{-\mathscr{H}[\varphi(x)] / T\}$, where $\mathscr{H}[\varphi(x)]$ is defined by (1). This correlator may be represented as an average with respect to the ground state of the Hamiltonian (4) of the $T$-product of Heisenberg operators

$$
\left\langle\mathrm{e}^{i Q \varphi(x)} \mathrm{e}^{-i Q \varphi(0)}\right\rangle=\left\langle 0\left|\hat{T}_{x} \mathrm{e}^{i Q \varphi(x)} \mathrm{e}^{-i Q \varphi(0)}\right| 0\right\rangle
$$

and it can be written as :

$$
\left\{\begin{aligned}
& \sum_{n} \exp {\left[-\frac{\left(E_{n}-E_{0}\right)}{T} x\right] \times } \\
& \times\left\langle 0\left|\mathrm{e}^{i Q \varphi}\right| n\right\rangle\left\langle n\left|\mathrm{e}^{-i Q \varphi}\right| 0\right\rangle, \quad x>0 \quad(17) \\
& \sum_{n} \exp \left[+\frac{\left(E_{n}-E_{0}\right)}{T} x\right] \times \\
& \quad \times\left\langle 0\left|\mathrm{e}^{-i Q \varphi}\right| n\right\rangle\left\langle n\left|\mathrm{e}^{i Q \varphi}\right| 0\right\rangle, \quad x<0 .
\end{aligned}\right.
$$

The matrix elements in (17) differ from zero only for states with the wave vectors $q_{n}=-(Q+n)$ at $x>0$ and $q_{n}=Q+n$ at $x<0$. We must substitute the values of the energies $E_{n}$ obtained above in these formulae. Note that for $m$ not too near to zero we can expand $E(q)$ in series over $q T$ even at $q \sim 1$ :

$$
E=E_{0}-i q T \frac{\partial E_{0}}{\partial h}+\frac{1}{2} q^{2} T^{2}\left|\frac{\partial^{2} E_{0}}{\partial h^{2}}\right|
$$

where $-\frac{\partial E_{0}}{\partial h}=\left\langle\varphi^{\prime}\right\rangle$ is given by (12) and $\left|\frac{\partial^{2} E_{0}}{\partial h^{2}}\right|$ is equal to $\frac{\partial}{\partial h}\left\langle\varphi^{\prime}\right\rangle$.

Using this formula we find :

$$
G_{Q}(x)=\mathrm{e}^{-i Q h x} \sum_{n} f_{n} \mathrm{e}^{i \alpha x-\beta|x|}
$$

where

$$
\alpha=\left\langle\varphi^{\prime}\right\rangle(Q+n), \quad \beta=\frac{1}{2} T\left(\frac{\partial}{\partial h}\left\langle\varphi^{\prime}\right\rangle\right)(Q+n)^{2}
$$

and

$$
\begin{aligned}
f_{n}=\operatorname{Re}\left\{\int_{0}^{2 \pi} \psi_{0}^{+}(\varphi)\right. & \mathrm{e}^{i Q \varphi} \psi_{-(Q+n)}(\varphi) \mathrm{d} \varphi \times \\
& \left.\times \int_{0}^{2 \pi} \psi_{Q+n}^{+} \mathrm{e}^{-i Q \varphi} \psi_{0} \mathrm{~d} \varphi\right\}
\end{aligned}
$$

$S(Q)$ will have sharp maxima at $Q$ near the reciprocal lattice vectors. Thus $Q=N+\Delta$, where $|\Delta| \ll 1$ and the structural factor (14) will take the form :

$$
S(Q)=\sum_{n} f_{n} \frac{\beta_{n}}{\left(Q a^{\prime}-N+n\left\langle\varphi^{\prime}\right\rangle\right)^{2}+\beta_{n}^{2}}
$$

where $a^{\prime}=1+\left\langle\varphi^{\prime}\right\rangle-h$ is a period of a deformed lattice divided by $2 \pi$. Thus, the neutron scattering spectrum consists of a number of sharp peaks, their positions being defined by the condition

$$
Q a^{\prime}=N-n\left\langle\varphi^{\prime}\right\rangle \text {. }
$$

This means that satellites are situated with the interval $\left\langle\varphi^{\prime}\right\rangle$. They are due to a superstructure of period $2 \pi /\left\langle\varphi^{\prime}\right\rangle$. From (12) we see that when $h$ tends to $h_{\mathrm{c}}$ the peak widths decrease, the satellites gradually merging. At $h \ll h_{\mathrm{c}}$ there are only almost $\delta$-functional maxima.

To have a notion of the coefficients $f_{n}$ we give their values for the case $N=1, h \geqslant h_{\mathrm{c}}, V(\varphi)=V \cos \varphi$, $n \neq-1$ :

$$
f_{n}=4\left(\frac{\pi}{K k}\right)^{4}(n+1)^{2} \frac{\exp \left[2(n+1) \pi \frac{K^{\prime}}{K}\right]}{\operatorname{sh}^{2}\left[2(n+1) \pi \frac{K^{\prime}}{K}\right]}
$$

where $K(k)$ is the complete elliptic integral of the first kind, $K^{\prime}=K\left(\sqrt{1-k^{2}}\right), k$ is the modulus of the elliptic function related with $h$ as :

$$
h=\frac{4 E(k)}{\pi k}
$$

$E(k)$ is the complete elliptic integral of the second kind. When $h \gg h_{\mathrm{c}}$ the modulus $k$ is small and

$$
f_{n}=(n+1)^{2}\left(\frac{k}{4}\right)^{8|n+1|-4 n}
$$

when $h \rightarrow h_{\mathrm{c}}, k \rightarrow 1$ and $f_{n}=(2 / K)^{2}$.

For $n=-1$ we obtain the following formula :

$$
f_{-1}=\left\{1+\frac{2}{k^{2}}\left[\frac{E(k)}{K(k)}-1\right]\right\}^{2} \text {. }
$$

When $k \rightarrow 0, f_{-1}=4(k / 4)^{4}$, at $k \rightarrow 1, f_{-1}=1$. The asymptotics of $f_{n}$ at $h \gg h_{\mathrm{c}}$ (i.e. when $k \rightarrow 0$ ) have been obtained in [13]. Let us point out that formula (21) is valid even at $h=h_{\mathrm{c}}$.

Thus we have completely investigated the behaviour of the system whose partition function is given by the path integral (3) with Hamiltonian (1). For any periodic potential $V(\varphi)$ and for an arbitrary initial incommensuracy $h$ we have found the free energy and the structural factor at low temperatures. We would like to emphasize that the Hamiltonian (1) not only describes a one-dimensional system of adsorbed atoms but other systems as well.

In conclusion we want to thank prof. V. L. Pokrovsky for his interest in our work and for useful discussions. We are also grateful to A. E. Lifshits. 


\section{References}

[1] Bol'shov, L. A., Napartovich, A. L., Naumovetz, A. G., FeDORUs, A. G., Usp. Fiz. Nauk 122 (1977) 125.

[2] Chinn, M. D., FaIn, S. C., Jr., Phys. Rev. Lett. 41 (1978) 955.

[3] Stephens, P. W., Heiney, P., Birgeneau, R. G., Horn, P. M., Phys. Rev. Lett. 43 (1979) 47.

[4] Bulaevsky, L. N., Khomsky, D. I., Zh. Eksp. Teor. Fiz. 74 (1978) 1863.

[5] Teodorou, G., Rice, T. M., Phys. Rev. B 18 (1978) 2840.

[6] Pokrovsky, V. L., Talapov, A. L., Zh. Eksp. Teor. Fiz. 75 (1978) 1151.

[7] Luther, A., Timonen, J., Pokrovsky, V. L., Preprint Nordita (1979).
[8] Bak, P., Mukamel, D., Villain, J., Wentowska, K., Phys. Rev. B 19 (1979) 1610.

[9] Pokrovsky, V. L., Talapov, A. L., Zh. Eksp. Teor. Fiz. 78 (1980) 269.

[10] Medvedev, V. K., YakivchuK, A. I., Ukr. Phys. J. 20 (1975) 1900.

[11] Okwamoto, Y., Takayama, H., Shiba, H., J. Phys. Soc. Japan 46 (1979) 1420.

[12] Brazovsky, S. A., Dzyaloshinsky, I. E., Obukhov, S. P., Zh. Eksp. Teor. Fiz. 72 (1977) 1550.

[13] Batistič, I., Barišič, S., J. Physique Lett. 40 (1979) L-613. 